\title{
Characterization of gamma irradiated plasticized carboxymethyl cellulose (CMC)/gum arabic (GA) polymer blends as absorbents for dyestuffs
}

\author{
SAYEDA M IBRAHIM*, ISSA M MOUSAA and MERVAT S IBRAHIM \\ Radiation Chemistry Department, National Centre for Radiation Research and Technology, Nasr City, Egypt
}

MS received 18 January 2013; revised 27 March 2013

\begin{abstract}
Polymer blends based on carboxymethylcellulose (CMC) and gum arabic (GA) were prepared by solution casting method. Glycerol was added to the polymer blend solution as a plasticizer with different ratios $(2.5,5,10$ and $20 \%)$. Then, the plasticized blends were exposed to gamma irradiation at different doses $(5,10$ and $20 \mathrm{kGy})$. The physical properties of the plasticized polymer blends were investigated in terms of gel fraction (\%) and swelling percent $(\%)$. Thermal properties were investigated by TGA. Also, the structure of the plasticized polymer blends was characterized by Fourier transform infrared spectroscopy. Scanning electron microscope was investigated in order to examine the compatibility between two polymers in the blend and also between polymer blend and plasticizer. The prepared plasticized polymer blends were used as an adsorbent for different dyestuffs. The sorption of dyestuffs by the plasticized polymer blend was determined by a method based on spectroscopic analysis. The results showed that the plasticized polymer blend has a high affinity for basic, acid, reactive and direct dyes. The obtained results showed that using glycerol as plasticizer improved the swellability of polymer blend and also the dye uptake (\%).
\end{abstract}

Keywords. CMC; GA; plasticizer; gamma radiation.

\section{Introduction}

Recent research and development of polymer materials have been directed to blending of different polymers to obtain new products having some of the desired properties of each component. Natural polymers are easily degradable and plentiful in resource and used widely in many industrial fields (Jianfa et al 2009).

Sorption by material of biological origin appears to offer a technically feasible and cost-effective approach to solve the problem of pollution (Volesky 2001).

Adsorbents, derived from natural polymers, such as cellulose, chitin, chitosan and their derivative have been identified as an attractive option because of their economic efficiency, distinctive properties, safety and biodegradability (Majeti and Kumar 2000; Isogai 2001). Carboxymethyl cellulose (CMC), the most popular and cheapest cellulose ether is well known as a safe and biodegradable material, which is widely used as food additives, washes, paste, etc. It is an anionic and water-soluble natural polymer derivative. It is widely used in detergents, oil exploration, food applications, paper and textile industries due to its high viscosity (Guo et al 1998). The addition of CMC to the cassava starch films increased tensile

\footnotetext{
*Author for correspondence (sayda.ibrahim@yahoo.com)
}

strength and reduced elongation at break of blended films. This was ascribed to the good interaction between cassava starch and CMC (Mbuna and Mhinizi 2003).

Gum arabic (GA) has been used in different purposes as in food, pharmaceutical, cosmetics and medical purposes (FAO 2000; Wirongrong et al 2011). Therefore, the necessity of using safe GA in food processing and medical purposes becomes more important. Ibrahim et al (2007) found that polyvinyl alcohol (PVA) polymer can be used as a modifier to improve the physical and mechanical properties of brittle GA polymer. Also, the presence of PVA in higher content protects the GA in the blend from degradation. The optimum ratio of GA in the blend was found to be $20 \%$ wt. These blends are very suitable to be used as natural material to release various antimicrobial drugs and also release the heavy metals to improve the agricultural soil.

CMC and GA have abundant hydroxyl groups in their structure hence on blending, hydrogen-bonding interactions between them are expected to occur. As a result, related thermal and mechanical properties could be improved (Ibrahim et al 2007).

In the present work, unirradiated and irradiated plasticized CMC/GA blends were prepared by adding glycerol at different concentrations to the blend as plasticizer. Different properties were investigated before and after exposure to various doses of gamma irradiation. The sorption 
capacity of CMC/GA blend for different dyestuffs was investigated.

\section{Experimental}

\subsection{Materials}

A sodium salt of CMC (pure polymer) in the form of granules, molecular weight (MW) 180,000 was supplied by El-Nasr Pharmaceutical Chemical-Prolabo (Egypt). GA is an exudate from Acacia species tree. GA is considered as heteropolysaccharides which contain more than one type of monosaccharide in complex branched polysaccharides that contains galactose, rhmnose, glucouronic acid and arabinose residues, besides highly branched polymer of various monosaccharides (Anderson and Wang 1990). $N, N^{\prime}$-methylene bisacrylamide (MBAM) from Aldrich, Wisconsin, USA, was used as a crosslinking agent. Glycerol was of pure grade and obtained from (Prolabo, France). Four dyestuffs, belonging to different classes were used throughout this work. These dyes were remacryl blue (basic dye), supplied by Hoechst (Germany); Sandolan E-3GSL (acid dye), drimarene blue K-3GL (reactive dye) and solar orange (direct dye) supplied by Sandoz (Switzerland).

\subsection{Preparation of $C M C / G A$ film}

CMC/GA blends with different compositions (100\% $\mathrm{CMC}, 80 \% \mathrm{CMC}$ and $60 \% \mathrm{CMC}$ ) were prepared by the solution casting technique. $\mathrm{CMC}$ and GA powders were dissolved in distilled water at room temperature. About $0.1 \mathrm{wt} \%$ of MBAM as a cross-linking agent was added to the polymer solution. Then glycerol was added to the complete miscible solution as a plasticizer agent with a ratio of $(2 \cdot 5,5,10$ and $20 \%)$. The complete miscible solutions are then casted onto glass dishes to form films with a thickness of $\sim 0.2 \mathrm{~mm}$. The cast films were dried at room temperature for $24 \mathrm{~h}$ and then placed in a vacuum oven at $50{ }^{\circ} \mathrm{C}$ to remove the residual water.

\subsection{Gamma irradiation}

Irradiation process was carried out using a Co-60 $\gamma$ cell (made in India) at the National Centre for Radiation Research and Technology, Cairo, Egypt, with a dose rate $6.92 \mathrm{kGy} / \mathrm{h}$. Irradiation was carried out under an air atmosphere. The different polymer blends were exposed to different doses of $\gamma$-irradiation $(5,10$ and $20 \mathrm{kGy})$.

\subsection{Characterization of CMC/GA blends}

2.4a Determination of gel fraction (\%): A known weight $\left(W_{1}\right)$ of the irradiated blend was extracted by water in a soxhlet apparatus for $24 \mathrm{~h}$ and then dried to a constant weight in vacuum $\left(W_{2}\right)$. The soluble fraction $(S F)$ was determined according to the following equation:

$$
S F(\%)=\left(\left(W_{1}-W_{2}\right) / W_{1}\right) \times 100 .
$$

Thus GF (\%) was calculated as follows

$$
G F(\%)=(1-S F) \times 100 .
$$

2.4b Swelling (\%): A known dry weight of the insoluble part of the blend $\left(W_{1}\right)$ after calculation of the soluble fraction was immersed in distilled water at room temperature for $24 \mathrm{~h}$. The swollen part of the blend at equilibrium was weighed $\left(W_{2}\right)$.

The swelling percentage $(S W)$ is determined by:

$$
S W(\%)=\left(W_{2}-W_{1} / W_{1}\right) \times 100,
$$

where $W_{1}$ and $W_{2}$ represent the weight of the dry and wet samples, respectively.

2.4c FT-IR spectroscopic analysis: FT-IR spectrophotometer model Mattson 100, made by Unicam (UK) was used for FT-IR measurements over the range 500$4000 \mathrm{~cm}^{-1}$.

2.4d Thermogravimetric analysis: TGA studies were carried out using a TGA-30 apparatus (Shimadzu, Kyoto, Japan), at a heating rate of $10^{\circ} \mathrm{C} / \mathrm{min}$ in air, over a temperature range from room temperature to $600{ }^{\circ} \mathrm{C}$.

2.4e Scanning electron microscope: The surface morphology of CMC/GA blend was examined by SEM. The micrographs were taken with JSM-5400 instrument manufactured by Joel, Japan.

\subsection{Dye sorption measurements}

The percentage sorption of the different dyestuffs by CMC/GA was determined by a general procedure based on spectrophotometer analysis. In this regard, standard curves were first constructed, representing a relation between different known concentrations from each dye and the corresponding light absorption (El-Salmawi et al 2001). The light absorption of the basic, acid, reactive and direct dyes were measured at wavelengths of 660, 515, 590 and $470 \mathrm{~nm}$, respectively. The different expressions governing these relations were calculated to be as follows:

Light absorbance (mg/L) $=0.223 \times$ basic dye concentration,

Light absorbance $(\mathrm{mg} / \mathrm{L})=0 \cdot 026 \times$ acid dye concentration,

Light absorbance (mg/L) $=0.023 \times$ reactive dye concentration,

Light absorbance (mg/L) $=0 \cdot 046 \times$ direct dye concentration. 
In this procedure, a certain concentration from each dye under investigation $(20 \mathrm{mg} / \mathrm{L})$ was first dissolved in certain volume of boiled water solution. A constant weight of plasticized blend $(0.5 \mathrm{~g})$ was then immersed in different dye solutions at various lengths of time and then the light absorbance of the residual dye solution was measured. The percentage sorption by the plasticized blend was determined according to the following equation:

Dye sorption $(\%)=$ dye concentration on plasticized blend/initial dye concentration $\times 100$.

\section{Results and discussion}

\subsection{Gel fraction $(\%)$}

The gel fraction (\%) of irradiated 100\% CMC and GA/CMC polymer blends having different compositions at various irradiation doses was given in figure 1. It can be seen that the increase of GA content in the blend is accompanied with decrease in GF (\%) for the same irradiation dose. On the other hand, it can be observed that GF $(\%)$ of either $100 \% \mathrm{CMC}$ or any GA/CMC polymer blend increases with increasing irradiation dose up to $20 \mathrm{kGy}$ due to the occurrence of crosslinking. Moreover, it was found that at a fixed dose $100 \%$ CMC posses a high value of gel fraction (\%) than the blend. Furthermore, on increasing GA ratio in the blend is accompanied with a decrease in the gel fraction (\%) at the same dose. Finally, it can be concluded that there is a marked increase in the gel fraction $(\%)$ of irradiated polymer blends especially for blends rich in CMC.

This behaviour was observed on the addition of different concentrations of glycerol $(2 \cdot 5,5,10$ and 20\%) as a plasticizer on $80 \% \mathrm{CMC} / 20 \% \mathrm{GA}$. The results obtained

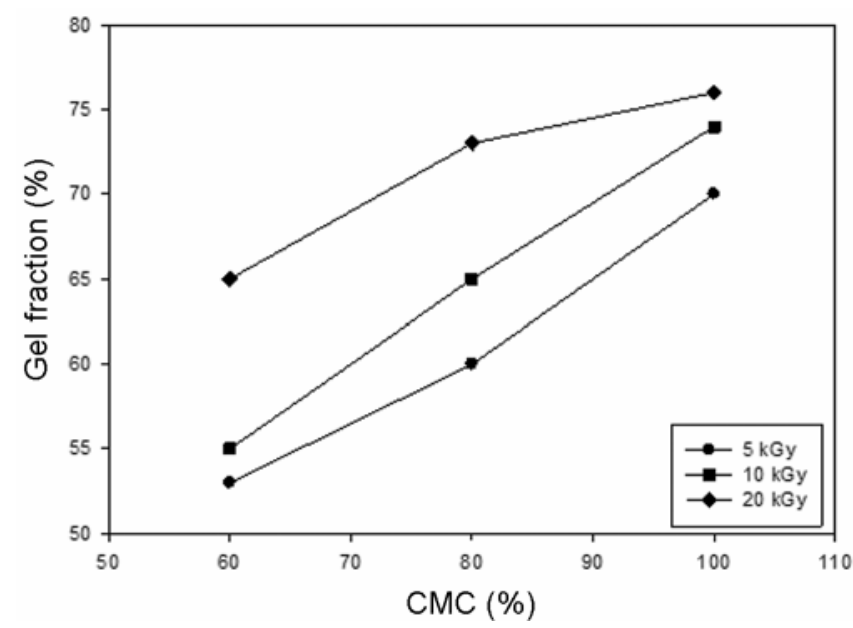

Figure 1. Effect of CMC \% in blend on gel fraction \% at different irradiation doses. are shown in figure 2 . It can be seen that, the increase of glycerol $(\%)$ is accompanied by a decrease in gel fraction for all irradiation doses under investigation. On the other hand, it can be observed that the gel fraction (\%) increases with increasing irradiation doses up to $20 \mathrm{kGy}$ for plasticized irradiated $80 \% \mathrm{CMC} / 20 \%$ GA blend.

\subsection{Swelling $(\%)$}

The swelling parameter is a very important factor for the applicability of the synthetic absorbent. Figure 3 shows effect of glycerol as a plasticizer on the swelling (\%) at different irradiation doses $(5,10$ and $20 \mathrm{kGy})$. It is clear that, the swelling $(\%)$ increases on increasing glycerol $(\%)$ in the blend at different irradiation doses. While increasing irradiation dose up to $20 \mathrm{kGy}$ is accompanied with decrease in swelling (\%). Thus, glycerol plays an

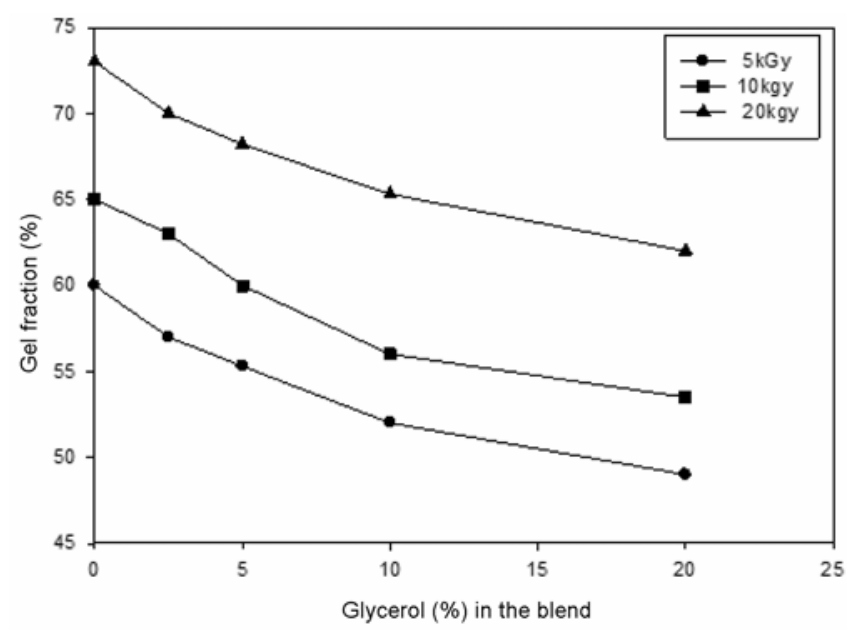

Figure 2. Effect of glycerol concentration in blend on gel fraction (\%) at different irradiation doses.

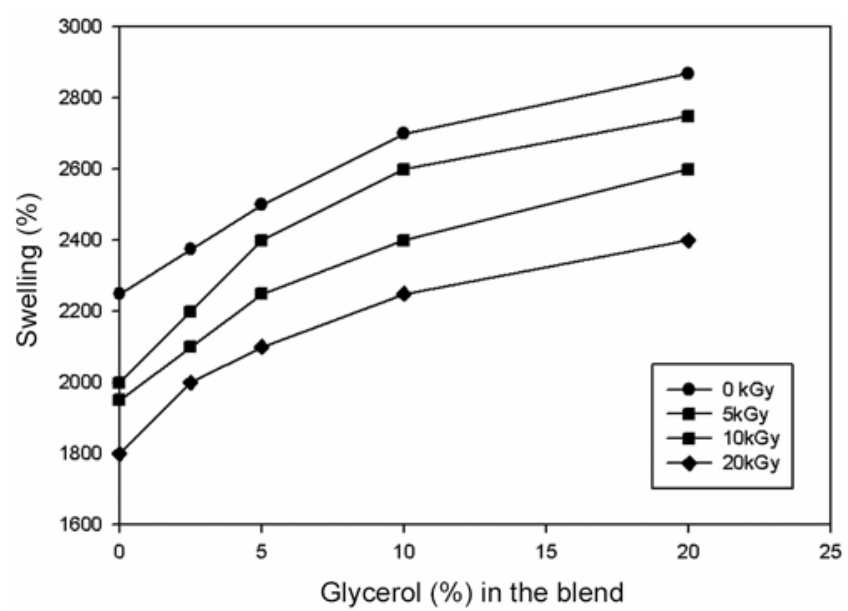

Figure 3. Effect of glycerol (\%) in blend on swelling (\%) at different irradiation doses. 
important role in plasticization of the blend which will be leading to increased swelling (\%). Also, the amount of absorbed water by the blend highly depends on irradiation dose. The relationship between water absorption and different irradiation doses was shown also in figure 3 . It can be seen that unirradiated blends have the highest swelling (\%) values than the irradiated samples. Moreover, the higher the irradiation dose, the lower the swelling (\%) this is due to the cross-linking density of $\mathrm{CMC} / \mathrm{GA}$ increased at higher doses.

\subsection{FT-IR spectroscopic analysis}

FT-IR spectra of plasticized unirradiated and irradiated (20 kGy) 100\% CMC and 80\% CMC/20\% GA blend were shown in figures 4 and 5 . In these figures, two strong peaks were observed at 1619 and $1420 \mathrm{~cm}^{-1}$ due to the asymmetrical and symmetrical stretching -COOgroups which appeared in unirradiated and irradiated

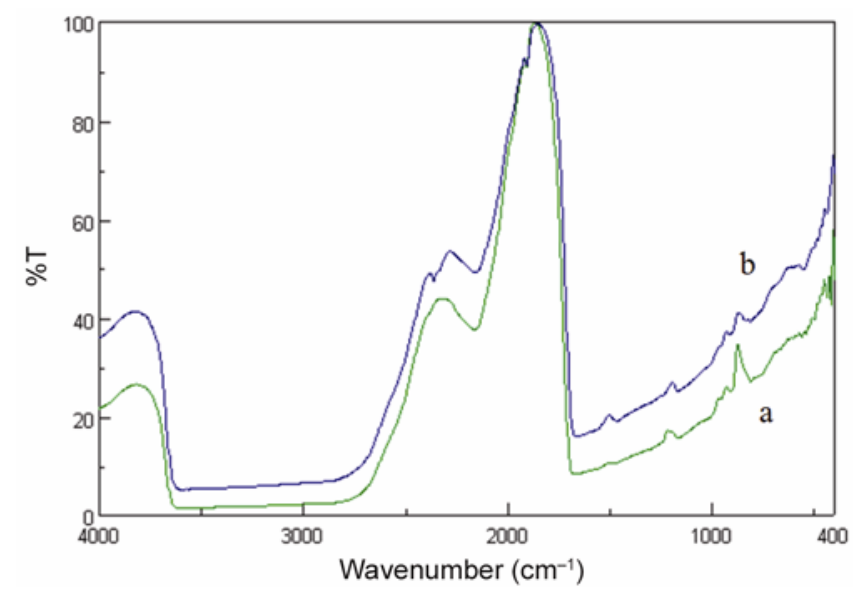

Figure 4. FT-IR spectrum of unirradiated plasticized (a) pure CMC and (b) $80 \% \mathrm{CMC} / 20 \% \mathrm{GA}$.

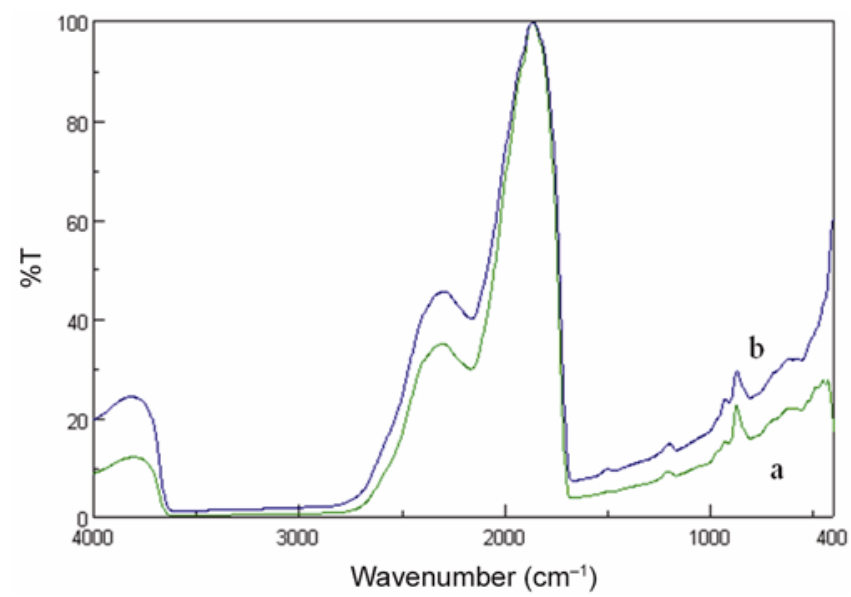

Figure 5. FT-IR spectrum of irradiated plasticized (20 kGy) (a) pure $\mathrm{CMC}$ and (b) $80 \% \mathrm{CMC} / 20 \% \mathrm{GA}$. spectra. Characteristic absorption peak of CMC and GA appeared at $3500 \mathrm{~cm}^{-1}$ for the hydroxyl groups (Silverstein and Webster 1998; Senna et al 2001). In the spectrum of unirradiated spectra there are bands at 2915, 1463 and $719 \mathrm{~cm}^{-1}$, responsible for deformed vibration of $\mathrm{CH}-$ groups (Ibrahim et al 2006) as shown in these figures. In these figures there was new bands around $1700 \mathrm{~cm}^{-1}$ due to $\mathrm{C}=\mathrm{O}$ absorption characteristic of $\mathrm{COOH}$ group of $100 \% \mathrm{CMC}$ and $80 \% \mathrm{CMC}$ in the unirradiated and irradiated spectra. However, it seems that polymer blend possesses relatively higher extent of hydrogen bonding than $100 \%$ CMC. Generally, slight changes in intensity for most characteristic band after irradiation at $20 \mathrm{kGy}$ was observed. It was found that there is a new and very weak peak appearing at $1.650 \mathrm{~cm}^{-1}$ that is attributed to the

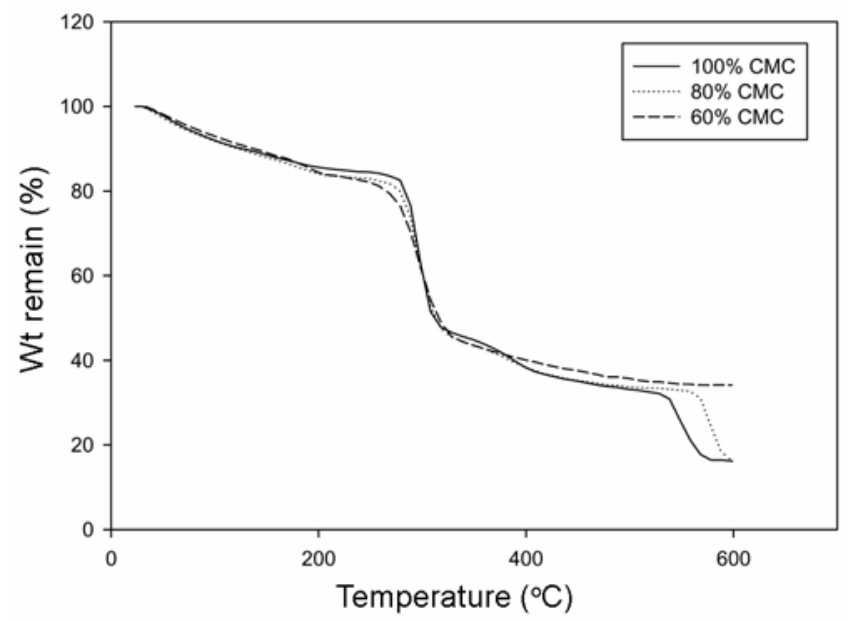

Figure 6. Initial TGA thermograms for thermal decomposition of unirradiated plasticized pure CMC and its blend with different ratios of GA.

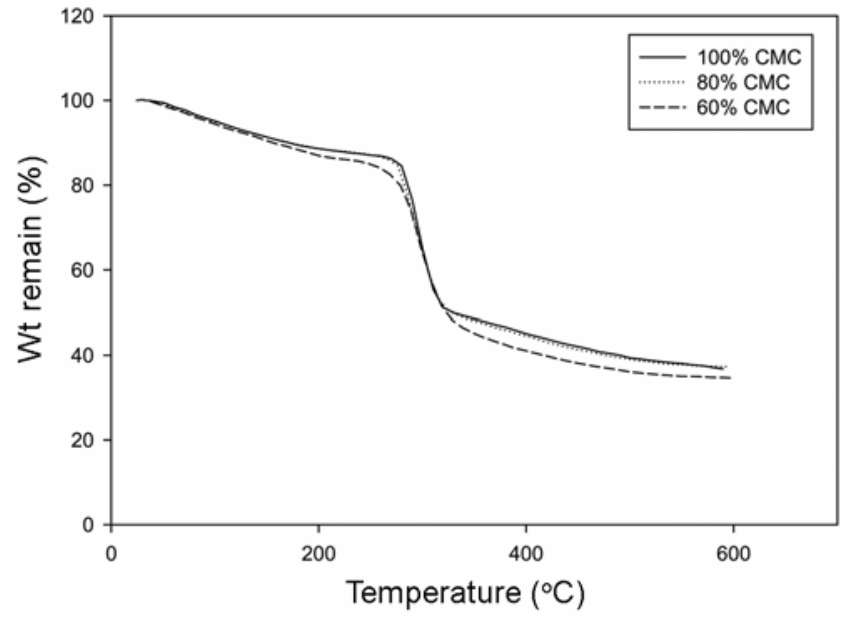

Figure 7. Initial TGA thermograms for thermal decomposition of plasticized irradiated pure $\mathrm{CMC}$ and its blend with different ratios of GA at a dose $20 \mathrm{kGy}$. 
presence of amide group of cross-linking agent (MBAM) in the blend.

\subsection{Thermogravimetric analysis}

The thermal stability of plasticized unirradiated and irradiated $100 \% \mathrm{CMC}, 80 \% \mathrm{CMC}$ and $60 \% \mathrm{CMC}$ within the temperature range up to $320^{\circ} \mathrm{C}$ are shown in figures 6 and 7. From these figures, it was found that there is an increase in thermal stability for irradiated than unirradiated polymer blends as shown in the figures. It is concluded that irradiation increases the thermal stability of both $100 \%$ $\mathrm{CMC}$ and its blend, which is due to the occurrence of a sort of cross-linking process due to irradiation as reported in another work (Senna et al 2001). Figure 8 was deduced from figures 6 and 7 which show dependence of thermal stability on a constant blend composition $(80 \% \mathrm{CMC} /$ $20 \%$ GA), which again confirm the increase of thermal stability on irradiation up to $20 \mathrm{kGy}$.

\subsection{Morphology by SEM}

Figure 9 shows structural surface morphology of plasticized unirradiated and irradiated 100\% CMC and its blend with different ratios of GA. It can be seen that the $100 \%$ CMC has smooth and relatively homogeneous appearance. After blending with GA a regular and parallel thin strains at blending ratios of plasticized $(80 \%$ $\mathrm{CMC} / 20 \% \mathrm{GA})$ and $(60 \% \mathrm{CMC} / 40 \% \mathrm{GA})$ as shown in the micrographs (b) and (c). On the other hand, the compatibility has appeared after irradiation at $20 \mathrm{kGy}$ where the molecular chains are compact and oriented parallel to each others as shown in micrographs (d), (e) and (f). The presence of glycerol in the blend is accompanied with an improvement in compatibility.

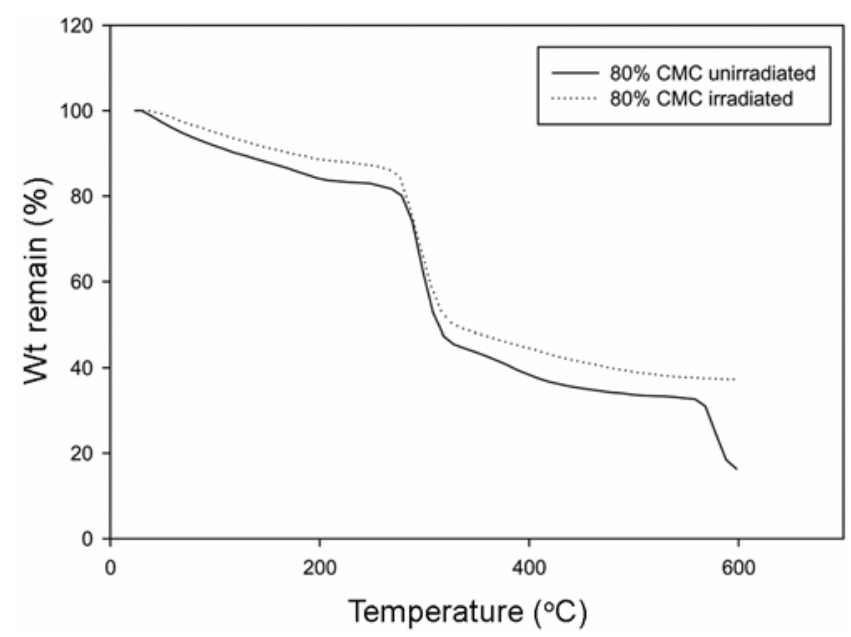

Figure 8. Initial TGA thermograms for thermal decomposition of plasticized $80 \% \mathrm{CMC} / 20 \%$ GA blend unirradiated and irradiated at $20 \mathrm{kGy}$.

\subsection{Sorption of dyestuffs by plasticized CMC/GA blend}

There are various types of dyes belonging to different classes depending on the kind of polymer blend to be dyed. For example, the reactive and direct dyes are specific for cotton cellulose fibres, while the acid and basic
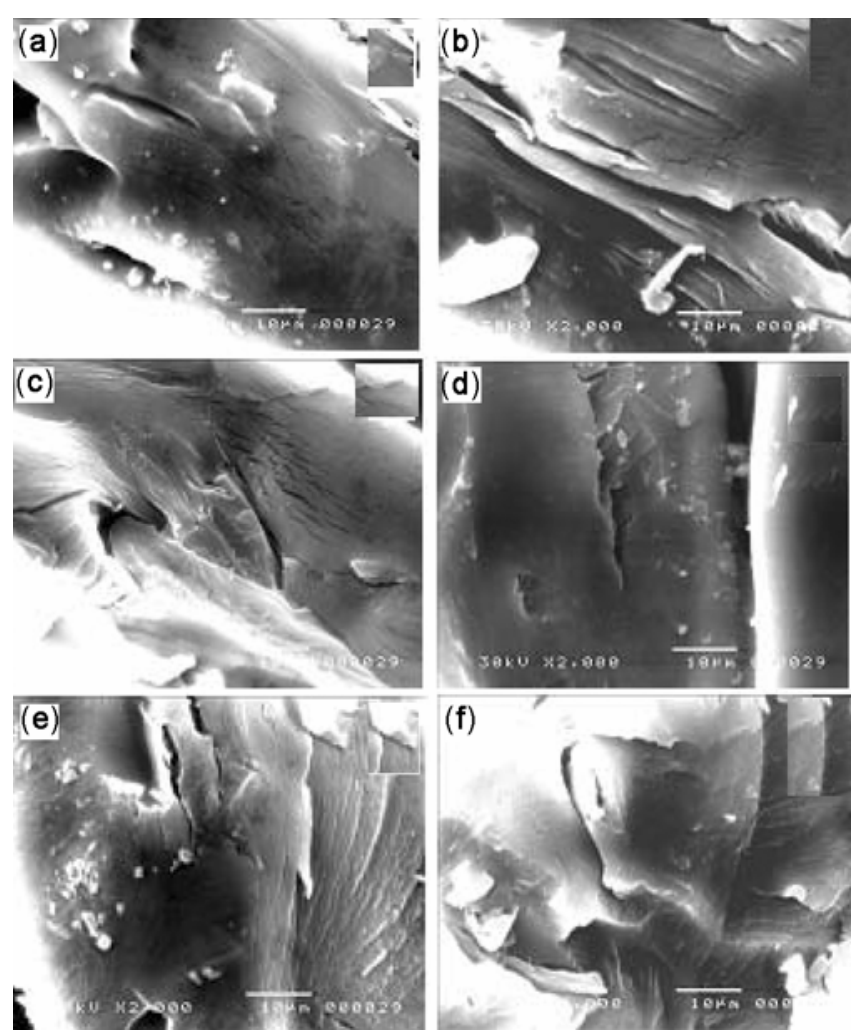

Figure 9. SEM micrographs of different unirradiated and irradiated polymer blends (a) unirradiated 100\% CMC, (b) unirradiated $80 \% \mathrm{CMC},(\mathbf{c})$ unirradiated $60 \% \mathrm{CMC}$, (d) $100 \% \mathrm{CMC}$ (20 kGy), (e) $80 \%$ CMC (20 kGy) and (f) 60\% CMC (20 kGy).

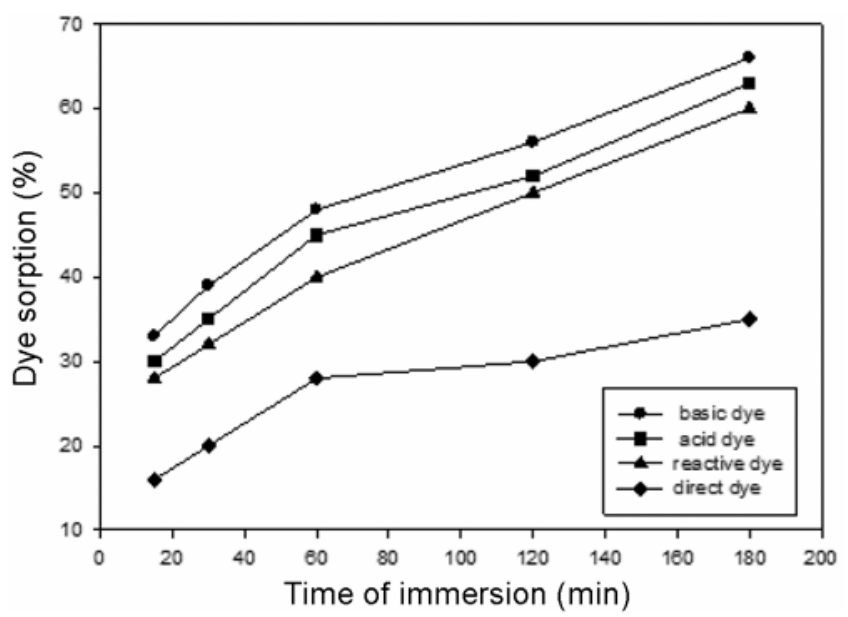

Figure 10. Dye sorption (\%) of basic acid, reactive and direct dyes by plasticized $80 \% \mathrm{CMC} / 20 \% \mathrm{GA}$ blend as a function of time. 
dyes are used for dyeing protein fibres such as wool and silk. In general, the choice of dyestuffs is based on the functional groups on both fibre and dyestuffs. All these dyes are usually used in textile factories.

In the present work, a solution containing a constant concentration of $20 \mathrm{mg} / \mathrm{L}$ of each dye under investigation was prepared. The dye sorption of different dyes by the plasticized $80 \% \mathrm{CMC} / 20 \% \mathrm{GA}$ polymer blends, as a function of the immersion time at room temperature at constant concentration of plasticizer $10 \%$ were investigated as shown in figure 10 . It can be seen that the dye sorption-time trend by plasticized $80 \% \mathrm{CMC} / 20 \% \mathrm{GA}$ blend differs from one dye to another. In all cases, the dye sorption was found to increase with an increasing time of immersion. The affinity of plasticized blend for the different dyes can be arranged as follows:

Basic dye $>$ acid dye $>$ reactive dye $>$ direct dye.

The affinity of CMC and GA for the different dyestuffs is essentially dependent on the hydrophilic group present in the polymer (hydroxyl group $\mathrm{OH}^{-}$). Also, the sorption process will eventually go through chemical bonding between the active groups on the different dyes and those of the blend and not through physical absorption. This is because, when the plasticized blend was left in the dye solution for a longer time than $120 \mathrm{~min}$, the adsorption of dyes did not occur. Thus, it may be concluded that dyeing of the plasticized blend with the different dyestuffs is dependent on the relative affinity for each dye. The mechanism of dyeing with basic dyes implies the presence of an anionic part in the medium to react with the cationic part of the dye through an anionic bond. Therefore, the sorption of the basic dye will go through the reaction with the hydroxyl groups of CMC and gum inside the blend.

\section{Conclusions}

The blending of already existing polymers is of special importance in the field of polymer chemistry. CMC and GA can form a thermodynamically miscible pair. The gel fraction increases with increasing irradiation dose, while swelling of plasticized CMC/GA blend nearly tends to increase with the increasing GA content and reduced with enhanced irradiation doses. Consequently, plasticized CMC/GA blends were selected to be used as an absorbent for dyestuffs.

\section{References}

Anderson D M and Wang W P 1990 Phytochemistry 291193

El-Salmawi K M, Abu Zaid M M, Ibrahim S M, El-Naggar A M and Zahran A H 2001 Appl. Polym. Sci. 82136

Food Agriculture organization (FAO) 2000 Satistical appendix (Sudan: IMF)

Guo J H, Skinner G W, Horcum W W and Barnum P E 1998 Pharm. Sci. Technol. Tod. 16254

Ibrahim S M, El-Salmawi K M and El-Naggar A A 2006 Appl. Polym. Sci. 1023240

Ibrahim M S, Ibrahim S M and Farag S A 2007 Polym. Plast. Tech. Eng. 461143

Isogai A 2001 Material science of cellulose (Tokyo: University of Tokyo Press)

Jianfa L, Jinhong L and Yimin L 2009 Appl. Poly. Sci. 112261

Majeti N V and Kumar R 2000 React. Funct. Polym. 461

Mbuna J J and Mhinizi G S 2003 Sci. Fo. Agric. 83142

Senna M M, Aly H A and El-Naggar A M 2001 Polym. Degrad. Stab. 7153

Silverstein R M and Webster F X 1998 Spectrometric identification of organic compounds (New York: Wiley) Vol. 6

Volesky B 2001 Hydrometallurgy 59203

Wirongrong T, Lisa J M, Sasitorn W, Pensiri S and Pornchai R 2011 Chem. Cent. J. 56 